\title{
Prognostic factors of intraoperative awakening
}

\begin{abstract}
Summary
A prospective longitudinal cohort study was carried out in patients announced for major elective surgery, with orotracheal general anesthesia, at the "Carlos Manuel de Céspedes de Bayamo Provincial Hospital, from the Cauto region during the period from January 1, 2016 until December 31, 2018; with the objective of identifying the risk factors hypothetically related to the prognosis of intraoperative awakening. The exposed cohort consisted of two patients who experienced intraoperative awakening in the study period, and met the inclusion criteria. The magnitude of the associations was estimated by calculating the relative risks (RR). Age equal to or greater than 65 years, difficult intubation, patients with ASA III and IV physical status, and chronic alcohol ingestion, were the surgical risk factors depending on the patient, associated with the prognosis of awakening. Intraoperative in patients announced for major elective surgery, under general orotracheal anesthesia, although not significantly; while the female sex did not present an association. The time of surgery equal to or greater than four hours, was constituted in the surgical risk factor depending on the surgery, related to the prognosis of appearance of intraoperative awakening in patients announced for major elective surgery, with orotracheal general anesthesia.
\end{abstract}

Volume I 3 Issue I - 202 I

\author{
Mg. Yacnira L Martínez-Bazán,' Dr. Alberto \\ Rodríguez- Carballo,' Yurisnel Ortiz- \\ Sánchez, ${ }^{2}$ Est. Sandra Blanco- Martínez,' Mg. \\ Bernardo Blanco-Zamora ${ }^{2}$ \\ 'Carlos Manuel de Céspedes General Teaching Hospital, Cuba \\ ${ }^{2}$ Faculty of Medical Sciences, Cuba
}

\begin{abstract}
Correspondence: Mg. Yacnira L. Martínez-Bazán, I Carlos Manuel de Céspedes General Teaching Hospital, July 26 \# 359 / Figueredo Y lora, Bayamo, Granma, Cuba, Tel 53234II 763, Email yacnibernar@gmail.com
\end{abstract}

Received: January 27, 2020 | Published: February 18, 2021

Keywords: Risk factors, intraoperative awakening, general anesthesia

\section{Introduction}

The objective of anesthesia is to induce unconsciousness through the administration of drugs and therefore, it is essential to provide the patient with analgesia, anxiolysis, amnesia and the suppression of hormonal, cardiocirculatory and motor responses to surgical stress. ${ }^{1}$ Measurement of anesthetic depth has always been a substantial necessity, even since the beginnings of ether anesthesia in $1847 .{ }^{1}$ Currently, the bispectral index (BIS) is the most frequently used anesthetic depth monitoring device. Its objective, based on a mathematical algorithm, measure the level of consciousness by means of the electroencephalogram (EEG) of the patient during general anesthesia, in order to evaluate its direct effects at the brain level. ${ }^{2}$ Waking up during anesthesia, with an intraoperative memory, occurs when the patient is able to process information and issue specific responses to various stimuli. ${ }^{2,3}$ The experience of consciousness is not the same in all patients, and can be grouped as memories (auditory perception, tactile sensation, sensation of paralysis and difficulty in moving and breathing, feeling of helplessness, panic, anxiety, chronic fear and fear of operation, insomnia and recurrent nightmares) ${ }^{4}$ and neurosis, known as post-traumatic stress disorder ${ }^{5}$ that requires psychiatric treatment

The risk factors for intraoperative awakening, according to epidemiological studies, can be classified into three main groups: Related to the patient, related to the type of operation and related to anesthetic technique. ${ }^{6}$ Justification of the investigation. The terror described by patients undergoing general anesthesia and affected by awakening in the intraoperative, is a unique fact for this patient. The unpredictable effect on your life and the long-term results are immeasurable. The fall in the incidence of awakening during anesthesia is connected to a better scientific and technical performance of the anesthesiologist, which involves issues such as understanding of the components of anesthetic activity, hypnotic drugs, analgesics, neuromuscular blockers, control of autonomic reflexes. and motors, in addition to the risk factors involved in this event. On the other hand, there is no epidemiological study of this phenomenon in the country, nor has it been systematically explored, it is not diagnosed if it is not searched because it happens that there are patients who do not confess their sufferings or are not aware of it as a defense mechanism or what happened more frequently, they share their concern with the surgeon and the surgeon, either due to the great care pressure that the surgery services have or due to the preponderant focus of medical attention on the cause for which the patient was operated on, the comment issued is ignored for the care that he presented an intraoperative awakening, as well as his traumatizing experiences during the development of the surgical intervention. The recognition of this complication as a potentially dangerous adverse event for the patient's health in the short and medium term and its conversion into a reason for complaints against the health services makes this condition an important entity, increasingly recognized in the environment current hospital and that forces us to have a better understanding of its complexity, its prevention and behavior in our environment; thus arising the following scientific problems: What are the risk factors hypothetically related to the prognosis of intraoperative awakening in patients announced for major elective surgery, under general orotracheal anesthesia? It is based on the hypothesis that female sex, age, difficult intubation, patients with ASA III and IV physical status, chronic alcohol intake and prolonged surgeries, constitute risk factors related to the prognosis of awakening. intraoperative in patients announced for major elective surgery, under general orotracheal anesthesia.

\section{Methodological design}

A prospective longitudinal cohort study was carried out in patients announced for major elective surgery, with general orotracheal anesthesia, at the Provincial Hospital "Carlos Manuel de Céspedes de Bayamo, from the Cauto region during the period from January 1, 2016 until December 31, 2018. The universe was made up of the 188 patients announced for major elective surgery, with general orotracheal anesthesia in the surgery service of the University Hospital "Carlos Manuel de Céspedes" of Bayamo, Granma, Cuba in the aforementioned period.

To calculate the definitive sample size, a prospective cohort study was designed. Starting from the universe of study, a probabilistic sample was taken and the selection of the cohort was carried out by 
simple random sampling. Establishing a prevalence of the exposure factor in the non-ill group of $33 \%$ and in the ill group of $23 \%$ with a $95 \%$ confidence coefficient that is equal to a 0.05 level of significance of the error, with a power of $80(1-\beta)$. It was established that the exposed cohort consisted of two patients who experienced intraoperative awakening in the study period and met the inclusion criteria. To calculate the sample size, the statistical package EpiInfo version 2002 for Windows was used. Selection of the exposed cohort: All patients older than 20 years of age, of both sexes announced for major elective surgery, with orotracheal general anesthesia, who express their consent to participate in the research in the general surgery room and who presented awakening, were consecutively included intraoperative.

Exclusion criteria for patients in the cohort.

$\neg$ Patients who are not willing to collaborate with the study.

$\neg$ Patients with cognitive disorders.

$\neg$ Patients announced for surgical interventions lasting less than one hour.

$\neg$ The patient already diagnosed refuses to carry out the survey to confirm intraoperative awakening Follow-up of patients in the cohort The anesthesia consultation will be carried out on the Friday before the week of the surgical intervention, where the patient will be thoroughly interrogated looking for signs of decompensation of their underlying disease that contraindicate the intervention; if necessary consult with the respective specialists according to underlying disease. Complementary exams necessary for intervention will be reviewed. Review medications that you are taking and maintain or withdraw according to the preoperative management protocol for these cases. A physical examination is performed by apparatus: taking blood pressure, heart rate, weight and height; everything will be reflected in anesthetic history. Indicate anesthesia, as well as premedication. Informed consent was explained and filled out In the salon, the patient was premedicated with $5 \mathrm{mg}$ diazepam, diluted intravenously, pre-oxygenated with $\mathrm{O}_{2}-100 \%$ through a face mask with spontaneous ventilation for five minutes, simultaneously non-invasive cardiovascular monitoring methods were established using the DoctusVII monitor. Anesthetic induction was carried out with the use of inducing drugs, but it was always performed with a general intravenous method and was supplemented with fentanyl. Relaxation was performed with the intravenous use of muscle relaxants; after being in stage 4, laryngoscopy was performed, stratifying all the laryngoscopies performed according to their degree of difficulty according to Cormack-Lehane Once the surgery was completed, a pharmacological reversal was performed if decurarization or denarcotization was necessary. He was transferred to the postanesthetic recovery room. Once the patient was awake in the recovery room and with full cognitive faculties, a neurological examination was performed in order to confirm the normality of the patient's state of consciousness and avoid biases caused by incomplete recovery from anesthesia, as well as dissociative states. This quick, but necessary neurological examination will consist of 6 elementary items to evaluate the superior cortical functions such as: time, space, person, memory and attention, added to the exploration of the pupillary reflexes. After confirming the normality of the state of the higher functions, the first survey was carried out: the Brice questionnaire modified by Abouleish and Taylor. If the patient still in the post-anesthesia recovery room has not recovered or is intubated, it was waited, but within 24 hours postoperatively, if in that time he is not empowered to respond, he will leave the cohort. Otherwise, if recovery of consciousness is achieved in the immediate postoperative period while in the intensive care room or in an open room, the Brice questionnaire modified by Abouleish and Taylor will be carried out. A second interview was conducted with the Brice questionnaire modified by Abouleish and Taylor, on the seventh day after general anesthesia, this can be carried out by the corresponding anesthesiologist or a nurse either in person or by telephone, as part of a routine postoperative evaluation. If a positive response is obtained in any of the interviews carried out, it was assumed as suspicion of intraoperative awakening during general anesthesia. Classification of intraoperative awakening was performed according to the Michigan classification instrument. All the data were also collected in the data collection sheet prepared for the study the evaluation of the diagnosis of intraoperative awakening was carried out during the entire surgical procedure and after it, and usually by two specialists in Anesthesiology and Resuscitation in unison. The primary data collection was obtained from the medical records of the patients operated on in the general surgery room of the hospital participating in the research, was carried out by two separate specialists and then the data were compared.

\section{Statistic analysis}

It was carried out in 3 stages: Descriptive study: The qualitative variables were represented by number and percentage and for the quantitative variables, measures of central tendency were obtained, such as the mean, median, as well as dispersion measures of the type of standard deviation (SD). Univariate analysis: The possible association between the variables considered in the study was studied. To assess the association between the variables, the Mantel Chisquare test was used. The magnitude of the associations was estimated by calculating the relative risks (RR) of DIO. Point estimates and by confidence interval (95\%) of the RRs were obtained. As part of the univariate analysis, the means of the quantitative variables were compared between those who did not experience OID and those who experienced OID. The statistic used for this purpose was the Student's $\mathrm{t}$ for normally distributed variables, the non-parametric Mann Whitney $\mathrm{U}$ test was used when a distribution other than normal was observed. Multivariate analysis: it consisted of fitting a logistic regression model with all the variables that were significant in the univariate analysis. The adjustment of the logistic regression function, which is equivalent to the estimation of its parameters, was performed by the maximum likelihood method. The Hosmer-Lemeshow goodness of fit statistic was also applied to evaluate the goodness of fit of the model. The SPSS version 21.0 statistical package was used for the entire analysis.

\section{Results}

Table 1 shows that difficult intubation was constituted in the study sample as a risk factor for experiencing intraoperative awakening when OR values greater than one were obtained (OR 61.00; 95\% CI: $0.77-4788,3 ; p=0.4918)$; however the results were not statistically significant. Regarding chronic alcohol consumption, although the risk of experiencing intraoperative awakening in the patient increased, the results were not statistically significant (OR 30.00; 95\% CI: 0.38 $2354.9 ; p=0.4833$ ). Similar results were obtained for the variable type of patient according to the classification system used by the American Society of Anesthesiology (OR 25.57; 95\% CI: 0.32-2007.2; p = $0,4804)$. On the other hand, age equal to or greater than 65 years increased the risk of experiencing intraoperative awakening (OR 7.45; 95\% CI: $0.29-585.15 ; p=0.4329$ ). (Results shown in table 1 ). In the 
study sample, the female sex was not a risk factor for experiencing intraoperative awakening as OR values lower than one were obtained (OR 0.55; 95\% CI: 0.007-43.17; $\mathrm{p}=0.2903$ ).

The surgical time greater than 4 hours was constituted in the investigation into the surgical risk factor based on the surgery associated with the experimentation of intraoperative awakening, when obtaining OR values greater than one (OR 4.81; 95\% CI: 0 ,
06-377.7; $\mathrm{p}=0.3961)$; however the results were not statistically significant (Table 2).

When the logistic regression model (Table 3 ) is adjusted to the data, in order to evaluate the independent value of the different risk factors, the number of factors was reduced to two, where it was observed that the risk factor of greater independence was difficult airway followed by alcohol consumption, all significantly $(\mathrm{p}=0.000)$.

Table I Surgical risk factors depending on the patient. Hospital Carlos Manuel de Céspedes, January 2016 to December 2018

\begin{tabular}{|c|c|c|c|c|c|c|c|c|c|c|}
\hline \multirow{2}{*}{ FR } & & \multicolumn{2}{|c|}{ With IA } & \multicolumn{2}{|c|}{ Without IA } & \multicolumn{2}{|c|}{ Total } & \multirow{2}{*}{$\mathbf{R R}$} & \multirow{2}{*}{ IC (95 \%) } & \multirow{2}{*}{$\mathbf{p}$} \\
\hline & & No & $\%$ & No & $\%$ & No & $\%$ & & & \\
\hline \multirow{2}{*}{ Sex } & Fem & I & 0,53 & 120 & 63,83 & 121 & 64,36 & \multirow{2}{*}{0,55} & \multirow{2}{*}{$0,007-43,17$} & \multirow{2}{*}{0,2903} \\
\hline & Mas & I & 0,53 & 66 & 35,11 & 67 & 35,64 & & & \\
\hline \multirow{2}{*}{ Age } & $\geq 65$ years & I & 0,53 & 22 & $\mathrm{II}, 70$ & 23 & 12,23 & \multirow{2}{*}{7,45} & \multirow{2}{*}{$0,29-585,15$} & \multirow{2}{*}{0,4329} \\
\hline & $<65$ years & I & 0,53 & 164 & 87,23 & 165 & 87,77 & & & \\
\hline \multirow{2}{*}{$\begin{array}{l}\text { Difficult intuba } \\
\text { tion }\end{array}$} & Yes & I & 0,53 & 3 & 1,60 & 4 & 2,13 & \multirow{2}{*}{61,00} & \multirow{2}{*}{$0,77-4788,3$} & \multirow{2}{*}{0,4918} \\
\hline & No & I & 0,53 & 183 & 97,34 & 184 & 97,87 & & & \\
\hline \multirow{2}{*}{ ASA } & III-IV & I & 0,53 & 7 & 3,72 & 8 & 4,26 & \multirow{2}{*}{25,57} & \multirow{2}{*}{$0,32-2007,2$} & \multirow{2}{*}{0,4804} \\
\hline & I-II & I & 0,53 & 179 & 95,21 & 180 & 95,74 & & & \\
\hline \multirow{2}{*}{ Alcoholism } & Yes & I & 0,53 & 6 & 3,19 & 7 & 3,72 & \multirow{2}{*}{30,00} & \multirow{2}{*}{$0,38-2354,9$} & \multirow[t]{2}{*}{0,4833} \\
\hline & No & I & 0,53 & 180 & 95,74 & 181 & 96,28 & & & \\
\hline
\end{tabular}

Origin: Survey.

Table 2 Surgical risk factors depending on the surgery. Hospital Carlos Manuel de Céspedes, January 2016 to December 2018

\begin{tabular}{|c|c|c|c|c|c|c|c|c|c|c|}
\hline \multirow{2}{*}{ FR } & & \multicolumn{2}{|c|}{ With IA } & \multicolumn{2}{|c|}{ Without IA } & \multicolumn{2}{|c|}{ Total } & \multirow{2}{*}{$\mathbf{R} \mathbf{R}$} & \multirow{2}{*}{ IC (95 \%) } & \multirow{2}{*}{$\mathbf{p}$} \\
\hline & & No & $\%$ & No & $\%$ & No & $\%$ & & & \\
\hline \multirow{2}{*}{$\begin{array}{l}\text { Surgery } \\
\text { time }\end{array}$} & $\geq 4$ hours & I & 0,53 & 32 & 17,02 & 33 & 17,55 & \multirow{2}{*}{4,81} & \multirow{2}{*}{$0,06-377,7$} & \multirow{2}{*}{0,3961} \\
\hline & $<4$ hours & I & 0,53 & 154 & 81,91 & 155 & 82,45 & & & \\
\hline
\end{tabular}

Origin: Survey.

Table 3 Risk factors associated with the appearance of intraoperative awakening. Multivariate analysis. 20I6 - 20I8. Significance level 0.05

\begin{tabular}{|c|c|c|c|c|c|c|c|}
\hline \multicolumn{8}{|c|}{ Variables in the equation } \\
\hline & & B & E.T. & Wald & gl & Sig. & $\operatorname{Exp}(B)$ \\
\hline \multirow[t]{7}{*}{ Step la } & Age & $-I, 146$ & 1,257 & ,832 & 1 & ,362 & ,318 \\
\hline & Sex & ,908 & $\mathrm{I}, 788$ & ,258 & I &, 612 & 2,479 \\
\hline & BMI & $-I, 378$ & $\mathrm{I}, 093$ & 1,589 & 1 & ,207 &, 252 \\
\hline & ASA & $-1,016$ & 2,275 & , 199 & 1 & ,655 & ,362 \\
\hline & Alcoholism & 16,405 & $16,783,652$ &, 000 & I & ,999 & $1.33 E+10$ \\
\hline & Air way & 15,254 & $40,192,982$ &, 000 & $\mathrm{I}$ & 1,000 & $4,2 \mid 4,747,232$ \\
\hline & Constant & $-17,856$ & $43,556,480$ &, 000 & I & 1,000 &, 000 \\
\hline
\end{tabular}

aVariable (s) entered in step I:Age, Sex, BMI,ASA, Alcoholism, Airway.

$\mathrm{DIO}(\mathrm{IA})$, Despertar intraoperatorio (intraoperative awakening)

\section{Discussion}

The study of DIO is a problem of special relevance in anesthesia, due to its consequences for the patient and its possible medico-legal implications. ${ }^{7}$ The consequences of DIO for patients are very varied: some patients report the event with indifference, as an anecdotal fact;
Others, however, experience anguish and anxiety when recounting their lived experience (be it auditory, tactile or painful) as reflected in Schwender's works. ${ }^{8}$ There are patients who, despite having no memory of the DIO event, in the days after surgery experience recurrent symptoms of anxiety and nightmares, in relation to the event that occurred. ${ }^{9-12}$ Not all experiences of intraoperative awakening are 
the same or carry the same consequences: for the patient and the anesthesiologist, the most worrisome are those associated with having experienced pain during the intervention, since most patients who experienced pain suffer psychological sequelae, and the probability of developing PTSD appears to be closely related to previous experience of severe pain. ${ }^{13-16} \mathrm{In}$ the present investigation, what patients reported were voices and discomfort from the endotracheal had. In addition, longer-term surgeries and with more severe patients seem to be associated not only with a higher incidence of OID but also with a higher probability of developing PTSD. OID is often the consequence of insufficient depth in the anesthetic technique used or the use of anesthetic drugs in doses lower than those actually required by the patient. ${ }^{13-16}$ Likewise, there are a series of factors that condition its appearance, well related to the patient (sex, age, consumption of drugs, alcohol or psychotropic drugs, difficult airway, tolerance to the drugs administered, among others), with the type of surgical intervention ( urgent in patient, polytraumatized, cardiac surgery, obstetric anesthesia or procedures in neonates and children under 5 years old) or with the anesthetic technique (inhalation, total intravenous, use of neuromuscular relaxants, use of nitrous oxide, combined anesthesia or shallow anesthetic depth). ${ }^{6,11,17-19}$ As stated by Graham et al20, there are cases of patients with OID in which a predisposing cause could not be identified (up to $2.5 \%$ in some studies carried out) and even fraudulent claims (up to $4.2 \%$ in some series of cases of DIO). There were no gender differences in patients with OID, while studies indicate that the number of demands for intraoperative awakening is three times higher in women than in men, mainly because women recover more quickly from anesthesia, the cause It is not clear and could be due to gender pharmacokinetic differences and / or alterations in sensitivity to anesthetics. ${ }^{13-16}$ There are published works in which it is observed that women wake up faster than men after anesthesia with propofol and alfentanil. Likewise, the plasma levels of remifentanil necessary to attenuate the hemodynamic response to surgical stimulation are also higher in women than in men. It seems that these differences in the pharmacokinetics of the drugs in both sexes could affect the incidence of OID. ${ }^{6,11,17-19}$ However, there are recent studies that claim not to find statistically significant differences in the presence of OID between both sexes. ${ }^{20}$ A higher incidence of awakening in young patients during general anesthesia has been described, ${ }^{6,11,17-19}$ contrary to the results of this study, where the median age of patients with DIO was 55 years; These differences in occurrence could be attributed to the administration of superficial anesthesia in older patients with multiple comorbidities, however the study data prevent this inference from being made by statistical methods. In adult patients ( $>18$ years), no relationship is found between age and the presence or absence of OID. Twenty In the study carried out by Domino et al., ${ }^{21} 8 \%$ of the cases of DIO were related to the existence of a difficulty in intubation, since the manipulations of the airway and repeated laryngoscopies after having administered a single dose of anesthetic inducer, promote the appearance of the painting. In a recent study, Ghoneim ${ }^{22}$ reveals that only $4.5 \%$ of OID cases are attributable to difficult intubation and prolonged laryngoscopy. Given that the incidence of difficult intubation ranges between $4.5-7.5 \%$ of patients, Ghoneim even raises the fact that difficult intubation does not perform a prominent role as a risk factor for OID and concludes that the administration of supplementary doses of hypnotic in induction would prevent the appearance of OID in these cases. In the present investigation, difficult intubation became a risk factor for the appearance of OID, as suggested by current studies. ${ }^{23}$ There is an increased risk for intraoperative awakening in ASA III and IV patients, undergoing major surgery, given the hemodynamic instability that they may present during general anesthesia, which may lead to a decrease in anesthetic requirements, running the risk of superficiality anesthetic and DIO. ${ }^{6,11,17-19}$ Similar results were obtained in the present investigation. The multicenter study that Sebel and his collaborators carried out in 2004 in the USA, ${ }^{13}$ and to which we have already referred, concludes that there is an increased risk of suffering OID in those ASA III-V patients undergoing major surgery, probably due to the use of minor dose of anesthetic drugs and consequently to the performance of a more superficial anesthesia. In addition, there is a significant increase in OID episodes in patients with hemodynamic instability during surgery, in whom the administration of anesthetic drugs was decreased and even suspended to avoid its cardiovascular depressant effect. Chronic consumption of these substances is known to increase anesthetic requirements due to the development of tolerance to them. This puts these patients at increased risk for OID. Even being a chronic smoker seems to increase the risk of developing an episode of OID..$^{6,11,17-19}$ Similar results were obtained in the present investigation. A descriptive observational study where all patients undergoing surgery under balanced general anesthesia at the Hospital Universitario del Caribe in Cartagena, between July 2015 and February 2016, were evaluated; 304 patients who underwent various surgical procedures under balanced general anesthesia were identified, with an incidence of $3.3 \%{ }^{11}$ The sensations described by all patients with DIO were inability to move and a foreign body sensation in the throat. The median age of the patients with OID was 55 years. The distribution by gender was $58.9 \%$ women and $41.1 \%$ men, $83.2 \%$ were ASA 1 or 2, ASA 3 had $15.8 \%$ and 4 only $1.0 \%$. The history of alcohol consumption habits and previous DIO was presented in 5.3\%, emergency surgery was $30 \%$ in the DIO group and $6.8 \%$ in the group without OID, $\mathrm{p}=0.0340$. The most frequently found surgical specialty was general surgery with $58.2 \%$. The use of neuromuscular relaxants was thus distributed, atracurium with $85.5 \%$, and succinylcholine $11.2 \%$. The general occurrence of difficult orotracheal intubation was $3.3 \%$, being $20 \%$ in the OID group and $2.7 \%$ without OID, $\mathrm{p}=0.0339$. The use of the minimum alveolar concentration (MAC) $<0.5$ was in the groups with and without OID of $20 \%$ and $0.7 \%$ respectively, $p=$ 0.0001 . Hemodynamic instability was present in $44.7 \%$ of the patients, the most frequent hypotension being $33.5 \%$, followed by hypertension with $7.2 \%$ and bradycardia with $3.9 \%$; the latter occurred in $30 \%$ of the patients with DIO versus $3.1 \%$ of the group without the event, $\mathrm{p}=$ 0.0006.25 The level of anesthetic depth is subjective and difficult to determine. Some clinical signs used for this are the abolition of the palpebral reflex, the absence of response to the verbal command, and the absence of movement and hemodynamic monitoring. All of them are predictors of low correlation with the level of real anesthetic depth. Due to this, for some years there have been in the market anesthetic depth monitors to carry out a more effective intraoperative control of the electrical activity of the brain. Some of them are: the Bi Spectral Index (BIS), Auditory Evoked Potentials (AEP) monitor and Narcotrend. The difficulty of obtaining an objective and precise data of the anesthetic depth can cause an overdose of drugs or administration of subtherapeutic doses.,24,25 Premedication with amnesic drugs such as benzodiazepines or scopolamine is a standard method to reduce the probability of intraoperative awakening. Adequate doses of inducing agents should be administered with supplementary doses if airway management is difficult and there is no contraindication to using inhalational anesthetic agents, avoid muscle relaxants except when necessary, try to use a minimum alveolar concentration of between 0.8 and 1 anesthetic or higher if possible, anesthesia devices should be optimally maintained, proper function of the IV pump ensured with pressure/volume alarms, and anesthetic agents administered through a single line functional and specific intravenous. ${ }^{6,11,17-19}$ In addition, the use of a monitor should be considered to assess the depth of anesthesia. 
There is no single monitor that provides reliable and unambiguous information on anesthetic depth. Auditory evoked potentials and electroencephalogram wave processing technology of different formats can be used..$^{25}$ The purpose of any of these forms of ECG analysis is to estimate the level of hypnosis the patient is experiencing. Electrodes placed in the frontal and temporal area are processed on a computer to create a numerical representation of the level of sedation: lower numbers correspond to greater depth, while higher numbers are found in awake or slightly sedated patients. ${ }^{25}$ The Bispectral Index (BIS) monitor is the most widely used. It has a high economic cost and has been approved by the Food and Drug Administration (FDA). In a prospective study of 4,945 patients, it was shown that the use of BIS reduces the incidence of awareness by $77 \%$. 3 Myles et al, cited by Baltodano ${ }^{26}$ in a multicenter study, indicate a reduction of $82 \%$. If the BIS is less than 40, the patient is under deep anesthesia; if it is greater than 80 , the patient is under superficial anesthesia. When approaching a patient who has suffered the phenomenon of intraoperative awakening, her doubts should be heard, given timely explanations, provide general knowledge of the consequences that can occur and discuss its most feared consequence, PTSD

\section{Conclusion}

Age equal to or greater than 65 years, difficult intubation, patients with ASA III and IV physical status, and chronic alcohol intake were considered surgical risk factors depending on the patient, associated with the prognosis of the appearance of the intraoperative awakening in patients announced for major elective surgery, under general orotracheal anesthesia, although not significantly; while the female sex did not present an association.

The time of surgery equal to or greater than four hours, was constituted in the surgical risk factor depending on the surgery, related to the prognosis of appearance of intraoperative awakening in patients announced for major elective surgery, with orotracheal general anesthesia.

\section{Technical or financial aid}

None.

\section{Conflicts of interest}

None.

\section{References}

1. Walker EM, Bell M, Cook TM, et al. SNAP-1 investigator group. Patient reported outcome of adult perioperative anaesthesia in the United Kingdom: a cross-sectional observational study. British Journal of Anaesthesia. 2016;117(6):758-766.

2. Schuller PJ, Newell S, Strickland PA, et al. Response of bis-pectral index to neuromuscular block in awake volunteers. British Journal of Anaesthesia. 2015;115(Suppl. 1):i103.

3. Tasbihgou SR, Vogels MF, Absalom AR. Accidental awareness during general anaesthesia-a narrative review. Anaesthesia. 2018;73(1):112122.

4. Han DW. Do you believe that processed EEG helps to prevent intraoperative awareness?. Korean J Anesthesiol. 2018;71(6):427-429.

5. Catalá-Ripoll Jose Vicente, Hidalgo-Olivares Víctor Manuel, Monsalve-Naharro José Ángel, et al. Intraoperative awareness as an example of the influence of cardiac output on anesthetic dosing: case report. Rev colomb anestesiol. 2018;46(4): 341-344.
6. Nunes Rogean Rodrigues, Porto Victor Camarão, Miranda Vivianne Trevia, et al. Factores de riesgo para el despertar intraoperatorio. Rev Bras Anestesiol. 2012;62(3):369-374.

7. Mashour GA, Avidan MS. Intraoperative awareness: controversies and non-controversies. Br J Anaesth. 2015;115(Suppl 1):i20-i26.

8. Schwender D, Kunze-Kronawitter H, Dietrich P, et al. Conscious awareness during general anaesthesia: patients' perceptions, emotions, cognition and reactions. Br J Anaesth. 1998;80(2):133-139.

9. Poveda Martínez MJ. Eficacia del índice biespectral en la evaluación de la profundidad anestésica, en pacientes sometidos a cirugía electiva con anestesia general en el "Hospital Carlos Roberto Huembes" en el periodo Junio 2015- Enero 2016. Tesis. Universidad Nacional Autónoma de Nicaragua. Nicaragua, 2017.

10. Rojas Vivanco XY. Despertar intraoperatorio en pacientes con anestesia general en el hospital docente UTPL, durante julio a diciembre de 2011. Tesis. Universidad Técnica Particular de Lojas. Ecuador. 2014.

11. Florez Ballestas JE. Incidencia de despertar intraoperatorio y frecuencia de factores de riesgo en pacientes bajo anestesia general balanceada en el hospital universitario del Caribe. Tesis. Universidad de Cartagena. Cartagena de Indias, 2016.

12. Ambulkar RP, Agarwal V, Ranganathan P, et al. Awareness during general anesthesia: An Indian viewpoint. J Anaesthesiol Clin Pharmacol. 2016;32(4):453-457.

13. Whitlock EL, Rodebaugh TL, Hassett AL, et al. Psychological sequelae of surgery in a prospective cohort of patients from three intraoperative awareness prevention trials. Anesth Analg. 2015;120:87.

14. Villegas S, Suarez S, Owuor J, et al. Intraoperative awareness and experience with a ketamine-based anaesthesia package to support emergency and essential surgery when no anaesthetist is available. Afr $J$ Emerg Med. 2019;9(Suppl):S56-S60.

15. Lubke GH, Kerssens C, Phaf H, et al. Dependence of explicity and implicit memory on hypnotic state in trauma patients. Anesthesiology. 1999;90(3):670-80.

16. Yun W, Yun Y, Yong-hai S, et al. Investigation an analysis of incidence of awareness in patients undergoing cardiac surgery in Beijing, China. Chin Med J. 2005;118(14):1190-1194.

17. Schiff, ND. Altered consciousness. In: Winn R, ed. Youmans and Winn's Neurological Surgery. 7th ed. New York, NY: Elsevier Saunders;2016.

18. Naci L, Graham M, Owen AM, et al. Covert narrative capacity: mental life in patients thought to lack consciousness. Ann Clin Transl Neurol. 2017;4(1):61-70.

19. Graham M, Owen AM, Çipi K, et al. Minimizing the Harm of Accidental Awareness Under General Anesthesia: New Perspectives From Patients Misdiagnosed as Being in a Vegetative State. Anesth Analg. 2018;126(3):1073-1076.

20. Sebel PS, Bowdle TA, Ghoneim M et al. The incidente of awareness during Anesthesia: a Multicenter United States study. Anesth Analg. 2004;99(3):833-839.

21. Domino KB, Posner KL, Caplan RA, et al. Awareness during anesthesia: a closed claim analysis. Anesthesiology. 1999; 90(4):1053-1061.

22. Ghoneim MM, Block RI, Haffarnan M, et al. Awareness during anesthesia: risk factors, causes and sequelae: a review of reported cases in the literature. Anesth Analg. 2009;108(2):527-535.

23. Sanders RD, Gaskell A, Raz A, et al. Incidence of connected consciousness after tracheal intubation: a prospective, international, multicenter cohort study of the isolated forearm technique. Anesthesiology. 2017;126(2):214-222. 
24. Schuller PJ, Newell S, Strickland PA, et al. Response of bis-pectral index to neuromuscular block in awake volunteers. British Journal of Anaesthesia. 2015;115(Suppl 1):i103.

25. Han DW. Do you believe that processed EEG helps to prevent intraoperative awareness?. Korean J Anesthesiol. 2018;71(6):427-429.
26. Baltodano Loria A. Awareness o despertar intraoperatorio generalidades acerca de este fenómeno. Revista medica de costa rica y centroamerica 1xix. 2012;(600)15-19. 